under surface of the oval disk. The slots are shaped so that two continuous jet sheets are directed downwards and slightly inwards. When the engine is started the jets first build up the air cushion under the disk and then maintain the pressure difference between the cushion and the atmosphere. The pressure difference deflects the air curtains and they spill outwards at the ground or water surface (the pressure difference times the height above the ground must equal the radial rate of change of momentum), Two curtains are used for stability reasons. A third of the compressed air is bled off into two parallel propulsion ducts of rectangular crosssection which lie along and above the flat sides of the oval disk. Vanes at each end of each duct provide the necessary longitudinal and lateral control.

Because the weight that can be supported is dependent on the disk area while the power required is proportional to the peripheral length, the economy of a hovercraft should improve with size. A reasonably flat operational surface is required and early applications may well be over surfaces which existing vehicles find difficult, for example, marsh, snow, sand, ice and shallow rivers. Serious consideration has been given to a 400-ton car ferry capable of carrying eight hundred passengers and eighty cars across the channel at a speed of 90 knots. A careful detailed study of hovercraft economics suggests a cost of between 8 and 18 pence per (payload) ton (nautical) mile in the speed-range $70-170$ knots. Both cost and speed lie somewhere between the current values for ships and commercial aircraft. If the hovercraft can prove its worth with modest payloads over short distances, then serious thought could be given to developing a trans-ocean hovercraft capable of crossing the Atlantic in $24 \mathrm{hr}$. This new competitor could not appear within the next ten years, however, and one wonders what the level of ship and particularly aircraft fares will be by then. Nevertheless, the hovercraft has an extremely interesting future and one can only congratulate the inventor and the development engineers on their achievement and remark that this is one enterprise where the Old World leads the New.

These remarks are based on a paper "The Hovercraft-a New Concept in Maritime Transport", by P. R. Crewe and W. J. Eggington, read before the Royal Institution of Naval Architects on November 19,1959 .

J. L. StollerY

\title{
NEW DELHI SYMPOSIUM ON ALGAE
}

$\mathrm{P}$ OSSIBLY because algae are usually inconspicuous in the temperate regions in which most microbiologists receive their training, the dominant role which these organisms may have in soil and water in other climates is apt to be overlooked. In India, although much excellent work has been done on algae, this has largely followed morphological and systematic lines, and relatively little attention has been paid to their activities and possible economic importance. The symposium on "Algology", held in the Indian Agricultural Research Institute, New Delhi, during December 7-11, under the auspices of Unesco and the Indian Council of Agricultural Research, had the object of stimulating investigations on the more practical aspects of the subject. It was opened by Mr. S. K. Patil, Minister for Food and Agriculture, and was attended by experts from Japan, England, the Federal Republic of Germany, and Israel, as well as by delegates from all parts of India.

An introductory address by Dr. M. S. Randhawa, chairman of the organizing committee, outlined the development of algal studies and lines of research which may be of practical importance. The principal uses to which these organisms may be put are mentioned below, but it may be noted that in the tropics algal growth often needs controlling rather than encouraging $\rightarrow$ a matter which was not considered in any of the papers which followed. The growth of blue-green algae, which generally occurs on buildings during wet weather, not only renders them unsightly but also corrodes the stone-work and presents a problem of major economic importance. Algal growth may also interfere in the manufacture of salt, it may block canals and irrigation channels, and pollute fresh water.

The papers presented covered nearly every aspect of modern knowledge of the algae. Classical morphology was represented by papers on the comparative morphology of the Charophytes, by V. S. Sundaralingam, and on reproduction in red algae, by $M$. $S$. Balakrishnan, and by a lecture by M. O. P. Iyengar, describing some of the many interesting species which have been discovered in India. Recent advances in the study of the submicroscopic morphology of algae were reviewed by T. V. Desikachary, who also discussed the structure of diatom frustules in the light of his own electron microscope investigations. Papers on algal cytology, which is especially important for the understanding of life-cycles and for taxonomy, were given by C. S. P. Rao, Y. S. R. K. Sarma and Y. B. K. Chowdary.

One of the most important of recent advances in algal physiology has been the introduction by $H$. Tamiya and his associates of the technique of syn. chronous culture as a means of studying the metabolic changes occurring during the life-cycle. A paper by H. Tamiya summarized the main results obtained and dealt particularly with the role of sulphur in the processes of cell maturation and division. Studies on the mineral nutrition of a Selenastrum were described by $K$. V. Natarajan. In a lecture on the extracellular products of algae, G. E. Fogg presented evidence showing that the liberation of these is widespread and that enzymes are included among the very varied kinds of substances involved. These extracellular substances may be of ecological importance in various ways, as, for example, by forming chemical complexes with inorganic ions. The kinetics of the production of extracellular nitrogenous substances by a nitrogen-fixing Nostoc isolated from the root nodules of Trifolium alexandrinum was discussed by G. S. Venkataraman. In another paper on nitrogen fixation, G. E. Fogg emphasized the close intermeshing of this process with photosynthesis which occurs in blue-green algae. He also reported nitrogen fixation by Chlorogloea fritschii, evidently the first unicellular member of the group in which this property has been 
demonstrated unequivocally. A paper by A. Watanabe dealt with the importance of blue-green algae in rice-fields. Nitrogen-fixing blue-green algae are found almost ubiquitously throughout tropical. sub-tropical and temperate regions and evidently contribute materially to the fertility of many soils. It has been found possible to grow Tolypothrix tenuis, a particularly vigorous nitrogen-fixing species, on the large scale and to preserve it as a culture on porous gravel. Inoculation of paddy fields with this has resulted in appreciable and long-continued improvements in yields of rice.

H. Tamiya, H. von Witsch and A. M. Mayer each considered various aspects of the mass culture of microscopic algae such as Chlorella for use as animal or human food. The average yields of Chlorella grown on the large scale correspond to the production of $14,000 \mathrm{lb}$. of protein per acre per year, whereas the corresponding figures are $600 \mathrm{lb}$. for grass, $420 \mathrm{lb}$. for peanuts, $90 \mathrm{lb}$. for milk and $54 \mathrm{lb}$. for meat. The results of feeding tests demonstrate that the alga is of high nutritive value. Convincing evidenee of the feasibility of large-scale culture was given in a film of the work of the Japan Micro-algae Research Institute. This has culture ponds of about an acre in total area producing 12 tons dry weight of Chlorella a year, which is largely used as a source of growth factors for the commercial culture of Lactobacillus. As a means of producing material which can be used directly in human or animal nutrition the process is not yet an economic one; the estimated cost of decolorized algal powder is 1.12 dollars per $1 \mathrm{~b}$.considerably more than that of cheap protein sources such as soy beans $(0.06$ dollar per $\mathrm{lb}$.) or fish meal $(0.13$ dollar per lb.) but a little less than that of skim milk powder ( $1 \cdot 20$ dollars per $1 b$.). In arid regions this high cost may be offset by the high degree of water conservation which is possible with algal cultures as compared with conventional crops, but for more general use cheaper methods are essential. To suit Indian conditions, it would be desirable to simplify the process so that it might be operated by peasants, and to this end M. O. P. Iyengar suggested that an examination should be made of algae known to grow prolifically in Indian fresh waters and which might be harvested easily.
The cost of algal culture can be minimized by combining it with the photosynthetic treatment of sewage and organic wastes by the oxidation pond method. A paper submitted from the Sanitary Engineering Laboratory of the University of California gave a résumé of this process and reported that algae harvested from oxidation ponds by alum flocculation proved satisfactory as a protein source in the diet of lambs. Field studies carried out at the Engineering College, Guindy (Madras), on the use of algae in sewage disposal, described by A. Raman, indicate that the method can be used under Indian conditions.

In a general lecture on the use of algae as food, $\mathrm{H}$. Tamiya, besides describing the possibilities of Chlorella, gave an account of the use of soaweeds for this purpose in Japan. Among these, the most popular is Porphyra tenera, which is cultivated in shallow seas to the extent of 4,000-5,000 tons dry weight per annum and which can command prices of up to 8.00 dollars per $1 \mathrm{lb}$. The amounts of marine algae available around India are relatively small, but a paper by $\mathbf{F}$. Thivy showed that there are, nevertheless, possibilities for their use as food and described cottage industry methods for the preparation of seaweed meal and agar. The ecology and distribution of Indian marine algae were considered in papers by J. N. Misra and K. S. Srinivasan.

Phytoplankton ecology was discussed in four papers. V. P. Singh described detailed observations made over a year on two lakes in Uttar Pradesh, the plankton of which is dominated by Microcystis. M. T. Philipose and S. V. Ganapathi gave more general descriptions of Indian freshwater phytoplankton in relation to fisheries and water supply. The marine phytoplankton of the west coast of India was considered in special relation to fisheries by $\mathbf{R}$. Subrahmanyan. The indications are that the phytoplankton production could support a considerably higher rate of fishing than it does at present.

At the final session a number of recommendations were approved for the extension of algal research in India on a broad front with the ultimate object of making more practical use of these organisms.

G. E. FogG

\title{
EROSION OF METEORITES IN SPACE AND THE DENSITY OF INTERPLANETARY GAS
}

\author{
By DR. L. REIFFEL \\ Physics Division, Armour Research Foundation, Illinois Institute of Technology, Chicago
}

$\mathrm{T}$ THE etching rate, due to interaction with interplanetary gas, for the Sikhote-Alin meteorite fall has been estimated ${ }^{1}$ as $\leqslant 2 \times 10^{-7} \mathrm{~cm}$. $/ \mathrm{yr}$. from an exposure life-time of $5 \times 10^{8}$ years, as determined by argon-38/argon-39 age measurements. From this result, Whipple ${ }^{2}$ has suggested that the maximum possible proton density of interplanetary space in the vicinity of the Earth is as shown in column 3 of Table 1. Whipple assumes that meteoritic etching is due solely to sputtering from solar protons and uses normal incidence sputtering yields for protons on iron as follows : 0.5 atom/proton for $10,000 \mathrm{eV}$. or more, 0.2 at $500 \mathrm{eV}$. and $\sim 0.02$ at $100 \mathrm{eV}$.
In the actual calculation, these yields are doubled to account, approximately, for the greater yields which are manifest at smaller angles of incidence.

The purpose of this article is to point out that solar protons are probably not the major factor in doterm. ining etching rates from sputtering by gas clouds ejected from the Sun. Calculated upper limits on interplanetary gas densities and erosion rates near the Earth may be changed appreciably as a consequence of including sputtering by elements heavier than hydrogen, and are uncertain for other reasons as discussed below. 